\title{
Interrupções nas passagens de plantão de enfermagem na terapia intensiva: implicações na segurança do paciente
}

\author{
Interruptions in intensive care nursing shift handovers: patient safety implications \\ Interrupciones en los cambios del turno de enfermería en la terapia intensiva: implicaciones en la \\ seguridad del paciente
}

\author{
Jéssica Grativol Aguiar Dias de Oliveiral. Luana Ferreira de Almeida"; Leni Fagundes de Assis Hirabae"li; \\ Karla Biancha Silva de Andrade ${ }^{v}$; Claudia Maria da Silva Sáv; Vanessa Galdino de Paula ${ }^{\text {VI }}$
}

\begin{abstract}
RESUMO
Objetivo: identificar fatores intervenientes durante a passagem de plantão em uma unidade de terapia intensiva. Método: estudo transversal, descritivo, observacional, quantitativo, totalizando 522 passagens de plantão entre profissionais de enfermagem, realizado em uma unidade de terapia intensiva de um hospital universitário do município do Rio de Janeiro, entre fevereiro e junho de 2017 . Foi utilizado para coleta de dados, mediante a observação e registro dos pesquisadores, um check-list relacionado aos principais fatores que podem interferir na passagem de plantão. Os dados foram analisados por estatística descritiva simples. O projeto foi aprovado por Comitê de Ética em Pesquisa. Resultados: os fatores mais observados foram: toque dos alarmes (79,6\%), conversas paralelas (19,3\%) e baixo tom de voz do profissional que realizou a passagem de plantão (11,1\%). Conclusão: os fatores intervenientes identificados são considerados agravantes para a comunicação efetiva entre a equipe de enfermagem e representam riscos para a segurança do paciente. Descritores: Comunicação; enfermagem; unidade de terapia intensiva; segurança do paciente.
\end{abstract}

\section{ABSTRACT}

Objective: to identify factors intervening during shift handovers in an intensive care unit. Method: this cross-sectional, descriptive, observational, quantitative study of 522 shift handovers among nursing personnel was conducted at an intensive care unit of a university hospital in the municipality of Rio de Janeiro, between February and June 2017. Data were collected by observation and recording by researchers using a checklist for the main factors that may interfere with shift changes, and were analyzed by simple descriptive statistics. The study was approved by the research ethics committee. Results: the factors most observed were: alarms ringing (79.6\%), parallel conversations (19.3\%), and quiet voice of the professional handing over the shift (11.1\%). Conclusion: the intervening factors identified were considered to be factors impairing effective communication between the nursing team and aggravating risks to patient safety.

Descriptors: Communication; nursing; intensive care units; patient safety.

\section{RESUMEN}

Objetivo: identificar factores intervinientes durante el cambio de turno en una unidad de terapia intensiva. Método: estudio transversal, descriptivo, observacional, cuantitativo, totalizando 522 cambios de turno entre profesionales de enfermería, realizado en una unidad de terapia intensiva de un hospital universitario del municipio de Río de Janeiro, entre febrero y junio de 2017 . Se ha utilizado, para la recolección de datos, mediante la observación y el registro de los investigadores, un check-list relacionado a los principales factores que pueden interferir en el cambio de turno. Los datos se han analizado por estadística descriptiva simple. El proyecto fue aprobado por el Comité de Ética en Investigación. Resultados: los factores más observados fueron: las alarmas sonando (79,6\%), conversaciones paralelas $(19,3 \%)$ y bajo tono de voz del profesional que realizó el cambio de turno (11,1\%). Conclusión: los factores intervinientes identificados son considerados agravantes para la comunicación efectiva entre el equipo de enfermería y representan riesgos para la seguridad del paciente.

Descriptores: Comunicación; enfermería; unidades de cuidados intensivos; seguridad del paciente.

\section{INTRODUÇÃO}

O tema deste estudo está centrado na meta 2 do Programa Nacional de Segurança do Paciente, instituído pelo Ministério da Saúde no ano de 2014 - melhorar a comunicação entre profissionais de saúde. A Aliança Mundial para a Segurança do Paciente define segurança do paciente como a redução do risco de danos desnecessários associados ao cuidado em saúde ao mínimo aceitável ${ }^{1}$.

Na unidade de terapia intensiva (UTI), por ser um ambiente complexo e com grande fluxo de profissionais da saúde e de informações, é imprescindível que

Enfermeira. Especialista em terapia intensiva.Universidade do Estado do Rio de Janeiro. Brasil. E-mail: jessicagrativol@hotmail.com

"Enfermeira no Hospital Universitário Pedro Ernesto. Doutora. Professora Adjunta, Universidade do Estado do Rio de Janeiro. Brasil. E-mail: luana.almeida3011@gmail.com I"Especialista em terapia intensiva. Enfermeira no Hospital Universitário Pedro Ernesto, Universidade do Estado do Rio de Janeiro. Brasil. E-mail: leni.assis@ @hotmail.com voutora. Professora Adjunta, Universidade do Estado do Rio de Janeiro. Brasil. E-mail: karla.biancha@gmail.com

v'Mestre. Enfermeira no Hospital Universitário Pedro Ernesto, Universidade do Estado do Rio de Janeiro. Brasil. E-mail: enfclaudiamaria@gmail.com

v'Mestre em Enfermagem. Professora Assistente, Universidade do Estado do Rio de Janeiro. Brasil. E-mail: vangalpa@gmail.com 
a comunicação seja efetiva e não deixe lacunas ${ }^{2}$. Uma das principais ferramentas para a comunicação entre a equipe corresponde à passagem de plantão (PP), visto que este momento favorece a organização das informações sobre os pacientes e a gestão do cuidado durante a jornada de trabalho ${ }^{3}$ As informações repassadas entre os profissionais devem ser relevantes no planejamento de ações e de prevenção dos eventos adversos, ou seja, incidentes capazes de provocar danos à saúde. Com vistas à garantia de uma assistência mais segura, estes incidentes devem ser minimizados ${ }^{4,5}$.

Sabe-se que existem fatores capazes de interferir negativamente nas PP e transições de cuidado, como a interrupções de profissionais, o não uso de instrumentos padronizados, toque de alarmes, distrações entre os profissionais, conversas paralelas, baixo tom de voz do profissional que realiza a passagem e até mesmo as intercorrências durante a assistência ao paciente crítico. Estes fatores comprometem a comunicação entre os profissionais e podem contribuir para falhas nos cuidados e no planejamento de enfermagem, representando, assim, uma fragilidade no planejamento da assistência à saúde ${ }^{1}$.

Nesse sentido, este estudo objetivou identificar fatores intervenientes durante a PP em uma UTI de um hospital universitário do Rio de Janeiro. No Brasil, ainda são poucos os estudos que avaliam fatores que possam interferir na transferência dos cuidados, durante a PP. Identificar e divulgar esses resultados poderá apontar oportunidades de melhoria na assistência ao paciente crítico e auxiliar no planejamento de intervenções centradas na segurança do paciente.

\section{REVISÃO DE LITERATURA}

$\mathrm{Na}$ área da saúde, a comunicação ineficaz está entre as causas-raízes de mais de $70 \%$ dos erros na assistência. Devido aos problemas relacionados à segurança do paciente, em 2004, a Organização Mundial da Saúde (OMS) criou a Aliança Mundial para a Segurança do Paciente. Observa-se que a falta de processos de comunicação integrados entre os profissionais são fatores que contribuem para as falhas durante a realização de cuidados e implicam na segurança do paciente'.

No contexto da comunicação efetiva existem processos denominados: handoff, que nos hospitais são passagens ou episódios nos quais um paciente e as suas informações são trocadas de profissional para profissional de forma a garantir o mínimo de erro possível no tratamento do mesmo; e handover, processo que implica transferência de responsabilidade, pela complexidade do tipo de informação a ser transmitida e as características dos profissionais ${ }^{6}$. Quando existem barreiras que influenciam a qualidade do handover, diminui a qualidade dos serviços estando estes mais susceptíveis à ocorrência de erros ${ }^{7}$.

A segurança do paciente está relacionada com a qualidade da assistência. Em relação aos hospitais, cabe salientar a relevância da promoção da cultura de segurança, sendo esta compreendida como um comportamento individual e organizacional que busca continuamente estabelecer o compromisso com a promoção de práticas seguras, evitar eventos adversos e, por fim, garantir a qualidade dos serviços ${ }^{8,9}$. Em síntese, a segurança do paciente pode ser definida como o estabelecimento de medidas que visam à minimização dos riscos associados à assistência. Nessa linha, a cultura de segurança favorece o aprimoramento de práticas seguras, por meio de melhorias na comunicação, no trabalho em equipe e no compartilhamento de conhecimentos ${ }^{10}$

Uma pesquisa em três hospitais de ensino do Rio de Janeiro, identificou uma incidência de $7,6 \%$ de pacientes com eventos adversos, sendo $66,7 \%$ destes evitáveis. Esse contexto incentivou na última década a promoção de diferentes iniciativas para garantir cuidados de saúde mais seguros ${ }^{11}$.

No âmbito hospitalar a PP é considerada uma ferramenta de comunicação e organização do trabaIho da enfermagem. Acrescenta-se ainda, que este procedimento promove a identificação de problemas e necessidades para posterior análise situacional que permitirá uma reorientação do cuidado².

Os fatores capazes de interferir na PP, além dos anteriormente citados, como a quantidade excessiva ou reduzida de informações, omissão ou o repasse de dados incorretos, registros ilegíveis e incompletos, interrupções e distrações entre os membros da equipe comprometem a comunicação entre os profissionais de enfermagem e podem contribuir para falhas nos cuidados de enfermagem ${ }^{12}$.

\section{MÉTODO}

Estudo transversal, descritivo, observacional não participante, de abordagem quantitativa. A pesquisa foi realizada em uma UTI geral de um hospital universitário de grande porte no município do Rio de Janeiro. A unidade possui capacidade para dez leitos, admite indivíduos adultos, com perfil clínico e cirúrgico para observação e tratamento da saúde.

Neste setor, as PP ocorrem do serviço noturno para o diurno com início às $7 \mathrm{~h}$, e do serviço diurno para o noturno, às 19h. A unidade conta com seis equipes de enfermagem divididas em dois turnos: diurno e serviço noturno. No período do estudo, contava com 14 enfermeiros, sendo um chefe e um diarista, 41 técnicos de enfermagem, e 15 residentes de enfermagem, sendo 7 do primeiro ano e 8 do segundo ano.

As PP são realizadas com a equipe de enfermagem reunida no posto de enfermagem, o qual encontra-se próximo à porta de entrada e saída da unidade. Para a transmissão de informações durante esse momento, a unidade conta com impresso próprio, elaborado previamente pelos enfermeiros do setor estudado. 
Tal instrumento contempla informações como nome do paciente, diagnóstico médico, anotações da visita multiprofissional diária, procedimentos realizados e agendados, nível de consciência, intercorrências ocorridas com o paciente, medicações utilizadas, parâmetros ventilatórios, características e presença ou ausência de secreção traqueal, presença de função intestinal, registro da diurese e aspectos relacionados à pele.

Foram incluídas neste estudo as PP realizadas do serviço noturno para o diurno e vice e versa, realizadas por enfermeiros, técnicos e residentes de enfermagem do primeiro e segundo ano. Excluídas aquelas realizadas por docentes e acadêmicos de enfermagem.

Os dados foram coletados no período de fevereiro a junho de 2017, em dias intercalados e aleatórios. As observações foram guiadas através de um formulário no modelo check-list. Este foi dividido em duas partes: a primeira parte comum a todas PP da UTI, relacionada às informações gerais, tais como número de participantes no momento, categoria profissional, data, horário de início e de término da atividade e a segunda parte, com os principais fatores intervenientes observados, capazes de gerar interferências.

Os dados foram organizados e tabulados com a ajuda do programa ExcelR, analisados por estatística descritiva simples e demonstrados através de tabelas. Em acordo com a Resolução $n^{\circ} 466$ de 2012, do Conselho Nacional de Saúde, que prevê os aspectos éticos e legais para pesquisa em humanos, o projeto deste estudo foi submetido ao Comitê de Ética em Pesquisa da instituição e aprovado sob o número do parecer CAAE 64134016.0.0000.5259.

\section{RESULTAdos E Discussão}

Foram realizadas 60 observações, sendo 40 (80\%) dessas realizadas no período diurno e todas guiadas pelo formulário elaborado pelos pesquisadores, obtendo-se informações de 522 PP. O tempo médio de duração das PP foi de 21 minutos, com mediana de 19. A transmissão das informações foi realizada da seguinte forma: 53 (88\%) pelo enfermeiro plantonista, 6 (10\%) pelo enfermeiro plantonista junto aos residentes do segundo ano, e 1 (2\%) por residentes de primeiro e segundo ano.

Verificou-se que, em 510 (98\%) PP, foi utilizado o instrumento padronizado na UTI estudada; porém, em 458 (90\%) dessas, o mesmo foi relatado de modo incompleto para a equipe e subutilizado. Isto pode ter ocorrido, entre outros motivos, pelo seu preenchimento inadequado ou por consequência de fatores intervenientes como conversas, alarmes e interrupções de outros profissionais ${ }^{3}$. De fato, a transição do cuidado guiada por um instrumento melhora a comunicação entre os profissionais; logo, sua utilização é recomendada para a segurança do paciente.

A descrição parcial dos fatos e situações acerca dos pacientes críticos também pode ser explicada pela falta de tempo em relação à grande carga de trabalho demandada pela unidade estudada, em relação ao quantitativo de pessoal de enfermagem e/ou pela possível não valorização das informações contidas no atual instrumento de PP pelo profissional ${ }^{13}$. Possivelmente, os enfermeiros e/ou residentes nesse contexto, diante de tantos afazeres, priorizaram mais aspectos diretos e clínicos, relacionados à gravidade e assistência dos pacientes, tais como preparo e administração de medicamentos, cuidados com suporte ventilatório e alimentação por sondas, em detrimento dos registros de situações relacionadas aos pacientes, também importantes na comunicação entre equipes.

Entre os fatores que interferiram nas PP, observou-se em 416 (80\%) destas, o toque dos alarmes de monitores, bombas infusoras e ventiladores mecânicos, que não foram atendidos em 370 (89\%) dos casos. Tal fato contribuiu para a presença de ruídos na unidade, podendo ter acentuado falhas de comunicação entre profissionais de enfermagem, acerca das informações sobre os pacientes durante as PP.

Apesar de a equipe de saúde da UTI se preocupar com as questões relacionadas à segurança do paciente, percebe-se que ainda há reduzida compreensão da importância dos sistemas de alarmes, visto que, a maior parte deles foi negligenciada durante as observações. Neste contexto, uma importante questão a ser discutida relaciona-se à fadiga de alarmes, denominada como fenômeno que ocorre quando um número muito grande de alarmes encobre os clinicamente significativos, possibilitando que alarmes de relevância clínica sejam desabilitados, silenciados e ignorados pela equipe ${ }^{12}$.

Os alarmes são comuns em unidades de pacientes graves, pois há sistemas de monitorização que permitem acompanhamento contínuo dos doentes internados e a identificação mais rápida de possíveis alterações. O que pode representar um risco para os pacientes é o não atendimento a esses sinais sonoros e a inadequada intervenção no problema identificado ${ }^{14-18}$. As equipes de enfermagem observadas, de modo geral, apresentaram mínima resposta aos alarmes; tal comportamento pode se tornar um problema grave para a segurança do paciente $\mathrm{e}^{5-10}$.

Quanto aos demais fatores intervenientes nas PP observadas, verificou-se, em 101 (19\%) delas, conversas paralelas entre a equipe de enfermagem; em 59 (11\%), baixo tom de voz do relator que estava transmitindo as informações; em 31 (6\%), interrupções por outros profissionais como médicos, nutricionistas e fisioterapeutas; em 19 (4\%), falta de clareza e objetividade nas informações repassadas; em 15 (3\%), necessidade imediata de cuidado enfermagem, devido a intercorrências como parada cardiorrespiratória, preparo e administração de medicações de emergência e intervenções em situações de instabilidade hemodinâmica; em 13 (2\%), toque de telefone celular, conforme mostra a Tabela 1. 
TABELA 1: Fatores intervenientes na passagem de plantão. Rio de Janeiro, RJ, Brasil, 2016.

\begin{tabular}{lcc}
\hline Fatores intervenientes na comunicação & $\mathbf{f}$ & $\mathbf{\%}$ \\
\hline Toque de alarmes & 416 & 80 \\
Não atendimento aos alarmes & 370 & 89 \\
Conversas paralelas & 101 & 19 \\
Baixo tom de voz & 58 & 11 \\
Interrupções & 31 & 6 \\
Falta de clareza e objetividade nas & 19 & 4 \\
informações & 15 & 3 \\
Assistência durante a PP & 13 & 2 \\
Toque de telefone celular & & \\
\hline
\end{tabular}

No contexto investigado, no momento da PP, existe grande fluxo de profissionais de diversas áreas como médicos, nutricionistas, equipe de enfermagem, residentes e acadêmicos, equipe de limpeza, o que pode gerar dispersão na comunicação e ruídos na abertura das portas.

Estudos destacam também que o espaço insuficiente, que não comporte adequadamente toda a equipe, ausência dos profissionais, ruídos de aparelhos, porta da unidade aberta e com circulação de equipamentos, solicitações dos pacientes e assistência aos mesmos, superlotação da unidade e realização de procedimentos, são barreiras enfrentadas pela equipe de enfermagem e se traduzem em componentes negativos para a realização da PP, criando lacunas e dificultando a comunicação, podendo prejudicar a continuidade da assistência ${ }^{16-18}$.

\section{CONCLUSÃO}

Este estudo identificou como fatores intervenientes, durante as PP na UTI investigada, diversos motivos que podem comprometer a comunicação entre profissionais de enfermagem, tais como - ruídos de equipamentos, conversas paralelas, subutilização do impresso padronizado, o baixo tom de voz do relator/transmissor da informação, a falta de objetividade nas informações.

Os desafios para a comunicação, nesse contexto, apontam a necessidade de reforçar o uso adequado da tecnologia presente na unidade, estímulo da participação de toda a equipe de enfermagem que transmite e recebe as informações, o incentivo aos registros e a utilização de instrumentos padronizados para passagens de caso, entre outros. A comunicação efetiva, entre os profissionais, é um tema ainda a ser amplamente discutido, sobretudo em um ambiente como o da UTI, complexo tanto pela gravidade dos pacientes, quanto pelo uso da tecnologia leve e dura, visando ao atendimento seguro e de qualidade.

As limitações do estudo abrangeram as oscilações das dinâmicas da PP ocorridas na unidade investigada, durante o período de coleta de dados, e o objeto de estudo restrito à equipe de enfermagem.

\section{REFERÊNCIAS}

1. Gonçalves MI, Rocha PK, Anders JC, Kusahara DM, Tomazoni A. Comunicação e segurança do paciente na passagem de plantão em unidades de cuidados intensivos neonatais. Texto \& contexto enferm. (Online). 2016; 25(1): e2310014.

2. Rodriguez L, Oliveira EO, França CS, Andrade TRS, Pontes JS, Aguiar MC, et al. Mapeamento da passagem de plantão sob a ótica dos profissionais de enfermagem. Enferm. glob. 2013; 31(2): 239-31.

3. Empaire PP, Amara ACKB. O que todo intensivista deveria saber sobre a passagem de plantão na unidade de terapia intensiva. Rev. Bras. Ter. Intensiva. 2017; 29(2): 121-3.

4. Ministério da Saúde (Br). Agência Nacional de Vigilância Sanitária. Resolução - RDC n 36 de 25 de julho de 2013. Institui ações para a segurança do paciente em serviços de saúde e dá outras providências. Brasília (DF): ANVISA; 2013.

5. Silva LD. Segurança do paciente no contexto hospitalar. Rev. enferm. UERJ. 2012; 20(3): 291-2.

6. Cohen M, Hilligoss $B$. The published literature on handoffs in hospitals: deficiencies identified in an extensive review. Qual. Saf. Health Care, Ann Arbor. 2010; 1(1): 109-13.

7. Santos MCD, Andrade AG, Guimarães G, Gomes A. Comunicação em saúde e a segurança do doente: problemas e desafios. Rev. Port. Saúde Pública. 2010; 10 (1): 47-57.

8. Duarte, SCM, Stipp MAC, Silva MM, Oliveira FT. Eventos adversos e segurança na assistência de enfermagem. Rev. bras. enferm. (Online). 2015; 68(1): 144-54.

9. Souza RFF, Silva LD. Estudo exploratório das iniciativas acerca da segurança do paciente em hospitais do Rio de Janeiro. Rev. enferm. UERJ. 2014; 22(1):22-8.

10. Teodoro WR, Aquino LAM. Análise do processo de passagem de plantão em uma unidade de internação pediátrica. REME rev. min. enferm. 2010; 14(3): 316-26.

11. Gouvêa CSD, Tavassos C. Indicadores de segurança do paciente para hospitais de pacientes agudos: revisão sistemática. Cad. Saúde Pública (Online). 2010; 26(6): 1061-78.

12. Broca PV, Ferreira MA. Equipe deenfermageme comunicação: contribuições para o cuidado de enfermagem. Rev. bras. enferm. (Online). 2012; 65(1): 97-103.

13. Oliveira RM, Leitão MTA, Silva LMS, Figueiredo SF, Sampaio RL, Gondim MM. Estratégias para promover segurança do paciente: da identificação dos riscos às práticas baseadas em evidências. Esc. Anna Nery Rev. Enferm. 2014; 18(1): 122-9.

14. Franco AS, Bridi AC, Karam MA, Moreira APA, Andrade KBS, Silba RCL. Tempo estímulo-resposta aos alarmes do balão intraórtico: práticas para cuidado seguro. Rev. bras. enferm. (Online). 2017; 70(6): 1273-9.

15. Wong A, Malvestiti AA, Hafner MGS. Síndrome de Stevens-Johnson e necrólise epidérmica tóxica: uma revisão. AMB rev. Assoc. Med. Bras. 2016;62(5): 468-73

16. Pedreirinho A, Godinho H, Pinto M, Correia P, Mendes F, Marques MC. Alarma de fatiga en la seguridad del paciente: revisión sistemática. RIASE revista ibero-americana de salud y envejecimiento. 2016; 2(2):544-62.

17. Albuquerque AM, Barrionuevo EA. Passagem de plantão: otimizando a performance da equipe. In: Viana RAPP, Torre M. Enfermagem em terapia intensiva: práticas integrativas. São Paulo: Manole; 2017; 185-94.

18. Oliveira MC, Rocha RGM. Reflexão acerca da passagem de plantão: implicações na continuidade da assistência de enfermagem. Enfermagem Revista (Online). 2016; 19(2): 191-8. 